\title{
Comparative Toxicity Study of Chemical Pesticide and Biopesticide by Daphnia Bioassay
}

\author{
Amrita I. Kakka†, Mihir D. Herlekar and Shivani Awale \\ Department of Environmental Science, The Institute of Science, Dr. Homi Bhabha State University, Mumbai-400032, \\ Maharashtra, India \\ †Corresponding author: Amrita I. Kakka; amritakakka27@gmail.com
}

Nat. Env. \& Poll. Tech.

Website: www.neptjournal.com

Received: 12-06-2020

Revised: $04-08-2020$

Accepted: 27-08-2020

Key Words:

Neemark

Tafgor

$\mathrm{LC}_{50}$

Combination ratio treatment

Two-way ANOVA

Aquatic ecosystem

\begin{abstract}
A comparative study was conducted to evaluate the toxicity of a biopesticide and a chemical pesticide using Daphnia magna as a model aquatic faunal species. The primary survey revealed that Neemark and Tafgor are being commonly used by the farmers. The acute toxicity tests were conducted on Daphnia magna for two series of concentration ranges $100 \mathrm{ppm}$ to $1000 \mathrm{ppm}$ and $1000 \mathrm{ppm}$ to 4000 $\mathrm{ppm}$. The (Lethal Concentration) $\mathrm{LC}_{50}$ values for series 1 of Neemark and Tafgor were $522.86 \mathrm{ppm}$ and 439.46 ppm, respectively, whereas it was 1840.48 ppm and $1335.97 \mathrm{ppm}$, respectively for for Series 2. A significant difference in the mortality rates between Neemark and Tafgor in the concentration range of $1000 \mathrm{ppm}$ to $4000 \mathrm{ppm}$ was observed $(\mathrm{t}=2.483, \mathrm{p}<0.05)$. A combination treatment of Neemark and Tafgor in different proportions showed that the 2:1 v/v (Neemark: Tafgor) ratio showed the lowest toxicity with a $\mathrm{LC}_{50}$ value of $1067.78 \mathrm{ppm}$, suggesting its preferability in application on the field. Two-way ANOVA shows that the concentration of pesticides plays a significant role in the mortality of Daphnia $(F=19.729, p<0.05)$ and so does the combination ratio treatment $(F=7.166, p<0.05)$. These results suggest that these two factors along with the selection of a suitable plant-based pesticide play a critical role in the reduction in mortality rates of aquatic organisms.
\end{abstract}

\section{INTRODUCTION}

India primarily has an agro-based economy with an $18 \%$ contribution of agriculture to the total GDP (Bharadwaj \& Sharma 2013, Kekane 2013). To sustain such a large economy, the longevity of crops and their resistance to myriad pests becomes a fundamental necessity. Pesticides play a vital role in maintaining world food production. Any substance that intends to prevent, destroy, repel or lessen the damage of any pest is called a pesticide (Eldridge 2008). There have been estimates that crop losses to pests would increase by $10 \%$ if no pesticides would be used at all (Pimentel et al. 1992). The most common categories of pesticides are insecticides, herbicides, fungicides, and rodenticides (Yadav et al. 2015). The type of pesticides used depends on the type of target pests.

The Green Revolution in India has resulted in the phenomenal growth in agricultural productivity by the use of high yielding varieties, chemical fertilizers, and pesticides (Kumar 2012). The pesticide consumption in India has risen from 434 metric tonnes to $46,195.16$ metric tonnes during the period 1954 to 2000 (Bharadwaj \& Sharma 2013). In India, the total as well as per hectare consumption of pesticides increased significantly after 2009-10. The pesticide consumption was $0.29 \mathrm{~kg} / \mathrm{ha}$ during $2014-15$ which is nearly
50\% higher than the use during 2009-10 (Subash et al. 2017). Two main types of pesticides viz., chemical and biological, originating from the respective sources are used to fight the menace of pests targeting high economic value crops. Some of the chemical insecticides used are DDT (Dichloro Diphenyl Trichloroethane), BHC (Benzene hexachloride), Malathion, and Carbaryl (Yadav \& Devi 2017). Chemical pesticides are known to have several negative impacts on human as well as environmental health. Synthetic chemical pesticides are known to exist as residues in soil, water, food etc., which may, in turn, lead to phytotoxicity, physiological deformities, diseases, mortality, population changes, genetic disorders etc. in many living organisms. They may enter the food chain and coupled with bioaccumulation could cause grave consequences. The increased concerns about the environmental effects associated with the use of these synthetic chemical pesticides have led to the search for chemical extracts from various biological organisms having insecticidal properties (Kumar 2012). A promising alternative is the use of biopesticides. A biopesticide is a formulation derived from naturally occurring substances that control pest populations in an eco-friendly way and through non-toxic mechanisms. The sources of biopesticides could be microorganisms, plants or animals and could include living organisms, their products or 
their by-products which could be used for the management of pests. Nearly 700 biopesticide products are available globally with 175 registered biopesticide active ingredients (Kumar 2012). The share of biopesticide in the total crop protection worldwide is small with a value of about 3 billion dollars and accounts for just 5\% of the total crop protection market (Marrone 2014). The most commonly produced and used forms of biopesticides in India are neem-based compounds, Bacillus thuringensis, nuclear polyhedrosis virus (NPV) and Trichoderms (Khandpal 2014). Biopesticides derived from the entomo-pathogenic bacterium, Bacillus thuringiensis (Bt), form $90 \%$ of the total microbial biopesticides available (Kumar \& Singh 2015). The plant-based biopesticide derived from neem is eco-friendly and very effective against many species of arthropods, nematodes, and fungi (Kandpal 2014). The seeds of the neem tree (Azadirachta indica) contain the active ingredient Azadirachtin which is a natural antifeedant, insect growth regulator, and sterilant (Hummel et al. 2015).

Any pesticide used in agriculture can enter the adjoining water body through agricultural runoff and soil erosion, which could lead to adverse impacts not only on the water quality but also on the associated aquatic life. After the entry into the aquatic ecosystems, pesticides can cause fishery losses in many ways such as increased mortality, killing of susceptible fish fry, and elimination of essential fish foods, such as insects and other invertebrates (Pimentel et al. 1992). Chlorpyrifos, an organophosphate pesticide, being very toxic to fish has caused fish mortality in waterways adjacent to treated fields (United States Environmental Protection Agency [US EPA] 2000). Pesticides are known to cause serious problems because they are designed to kill organisms, both target and non-target ones (Hanazato 2000).

The toxicity of pesticides on an aquatic ecosystem can be assessed using different test organisms. The effects of two chemical pesticides viz. Imidacloprid and RH-5849 on earthworm (Eisenia foetida) were studied (Luo et al. 1999) in terms of their acute toxicity, effects on sperm morphological deformity, and biochemical toxicity. Some toxicity tests use fish such as Danio rerio as the test organism to determine the (lethal concentration) $\mathrm{LC}_{50-96 \mathrm{~h}}$ of neem-based pesticides (Maranho et al. 2014). Daphnia magna, a zooplankton, is also often used as the test organism. Since zooplanktons are very sensitive to toxic chemicals and occupy a central position in the lentic food chain, they are frequently used in ecotoxicological tests. The responses of zooplankton to toxicity tests provide information on the relative impacts on the ecosystem as a whole (Hanazato 2000). Daphnia has been used to test the effects of anthropogenic chemicals, such as dieldrin on the sex ratio and alteration in the male individuals' production (Dodson et al. 1999). They quantified the swimming behaviour of free-living Daphnia that were exposed to the pesticide. Two types of behaviours were seen: (1) 'spinning' (extreme and continuous escape behaviour) in response to acutely toxic levels of 'carbaryl' and (2) 'irritation' (an increase in escape-like behaviour) in response to sublethal levels. Daphnia magna has been used in acute toxicity test for neem-based biopesticides to determine the median Effective Concentration $\left(\mathrm{EC}_{50-48 \mathrm{~h}}\right)$. After these acute toxicity tests, chronic toxicity tests for 21 days were conducted (Maranho 2014). The moulting process of crustaceans has been interfered by azadirachtin, the active component in neem and some other aquatic invertebrates may also be susceptible (Stark 2007).

The objective of the present study was to generate a database on the most frequently used pesticides (chemical and/or biological) amongst a group of farmers of the Palghar district of Maharashtra, India. Subsequently, the toxicity testing of the most preferred biological pesticide and chemical pesticide was performed individually and in combination using Daphnia to ascertain the one, which is comparatively less harmful to the aquatic organism. This is the first of such an experimental analysis using the actual field concentrations of pesticides to assess their toxicity in terms of the $\mathrm{LC}_{50}$ values. Along with this, the combination ratio treatment experiments can provide a guideline to assess their potential use by farmers which could reduce the toxic effects on the aquatic ecosystem. The toxicity data so obtained were analysed statistically using the PAST software and MS Excel.

\section{MATERIALS AND METHODS}

The entire study was divided into two parts, wherein the first part included a questionnaire survey of farmers and the second half consisted of laboratory analysis of the toxicity of the most commonly used pesticides in the study area.

\section{Questionnaire Survey}

The questionnaire survey regarding the use of pesticides for agriculture was conducted among a group of 20 farmers in the Palghar district of Maharashtra. It comprised of a set of questions to collect data in terms of the type of crops cultivated, type of pesticide used (biological/ chemical), and the most preferred market-based pesticide product. Based on the questionnaire survey, the most preferred market products of biopesticide and chemical pesticide were found to be Neemark and Tafgor, respectively. These were chosen for the laboratory analysis to ascertain their respective $\mathrm{LC}_{50}$ values using the test aquatic organism Daphnia magna.

\section{$\mathbf{L C}_{50}$ Studies}

Stock solutions of Neemark and Tafgor of concentrations $500 \mathrm{ppm}$ and $2500 \mathrm{ppm}$, respectively were prepared. These 
concentrations were based on the volumes of pesticide used in the study sites by the farmers. Two series of diluted stock solutions of varying concentrations viz., 100 to $1000 \mathrm{ppm}$ with an interval of $100 \mathrm{pm}$ and $1000-4000 \mathrm{ppm}$ with an interval of $500 \mathrm{ppm}$ were prepared for both the pesticides. Thus, two concentrations range each for Neemark and Tafgor were prepared. This was done to accommodate both the pesticide concentrations used in the field.

Experiments were conducted to determine the lethal concentration of the pesticide that killed fifty percent of the population $\left(\mathrm{LC}_{50}\right)$ using probit analysis. The experimental work included two parts. The first part included finding the $\mathrm{LC}_{50}$ values for the individual pesticide range. In the second set of experiments, Daphnia was subjected to combination treatments of different volume: volume (v: v) ratios viz. 1:1, 1:2 and 2:1 of Neemark and Tafgor, respectively. This was done to determine the most favourable treatment that could be used by the farmers which are the least damaging to the aquatic fauna. In these combination treatment sets, varying concentrations of the two pesticides in the range of $100 \mathrm{ppm}$ to $500 \mathrm{ppm}$ were used to find the $\mathrm{LC}_{50}$ value of the particular combination treatment. All the experiments were initiated by transferring 15 live Daphnia into clean petri plates where each plate represented a particular concentration. Respective volumes of the pesticide concentrations from the series were inoculated in the plates. The number of dead Daphnia individuals was counted after an incubation time of $60 \mathrm{~min}$. All the experiments were carried out in duplicates. The percent mortality was calculated using the following formula presented in Equation 1.

\section{Percent Mortality $=($ Number of dead Daphnia $)$ Initial Number of live Daphnia) $\times 100$}

The percent mortality was calculated and transformed into probit values using Finney's probit table (Finney 1971). All the probit values were plotted against the log values of the concentrations. The log concentration value for the probit value 5 (representing fifty percent mortality) was found, the antilog of which yielded the lethal concentration that caused 50 percent mortality. The acute toxicity tests were conducted in a manner similar to the previous studies (Demetrio et al. 2014, Maranho et al. 2014) with a few changes to suit the present study.

Two-way ANOVA was done to estimate whether the different combination treatments and different pesticide concentrations used for the combination treatment affected the percent mortality significantly or not. The significant difference in the percent mortality between the different concentration ranges of Neemark and Tafgor was checked with the help of unpaired $\mathrm{t}$-test. The statistical analysis and graphical representations were done using PAST and MS Excel.

\section{RESULTS AND DISCUSSION}

\section{Questionnaire Survey}

The questionnaire survey conducted in the Palghar district of Maharashtra to understand the proportion of farmers using biopesticide and chemical pesticide revealed that out of the 20 farmers surveyed, $40 \%$ used only biological pest control measures, such as cow urine, Neemark and other neem products; $20 \%$ of them used only chemical pesticides such as Tafgor, Karathane, and Rogor, whereas $40 \%$ of the surveyed farmers used both chemical and biological pesticide. The different chemical and biological pesticides were used as a common application against all types of pests including aphids. The most common form of biopesticide and chemical pesticide used was Neemark (35\%) and Tafgor (55\%), respectively.

\section{$\mathrm{LC}_{50}$ of Pesticides}

Based on the results of the survey, Neemark and Tafgor were selected as the toxicants to carry out further acute toxicity tests. The median lethal concentration for Neemark for Series 1 (100-1000 ppm) and Series 2 (1000-4000 ppm) was found to be $522.86 \mathrm{ppm}$ and $1840.48 \mathrm{ppm}$, respectively. Various acute toxicity tests have been conducted on Daphnia using neem-based compounds, such as Neemix, Bioneem, and Margosan-O. In one of the studies, effective concentration $\left(\mathrm{EC}_{50}\right)(48 \mathrm{~h})$ of Margosan-O on Daphnia magna was as high as $125 \mathrm{ppm}$ as against the $\mathrm{LC}_{50}(48 \mathrm{~h})$ value of $13 \mathrm{ppm}$ (Scott \& Kaushik 1998). Another research found the $\mathrm{EC}_{50}$ for Margosan-O on Daphnia magna at 103 ppm (Saucke \& Schmutterer 1992). The $\mathrm{LC}_{50}(48 \mathrm{~h})$ of Neemix for Daphnia pulex was estimated to be 0.68 ppm by Stark (2001). He also found that the $\mathrm{LC}_{50}$ of Azatin for D. pulex was 0.57 ppm. Another acute toxicity test revealed that $\operatorname{LC}_{50}(48 \mathrm{~h})$ values for Neemix and Bioneem on Daphnia pulex were $0.028 \mu \mathrm{g} / \mathrm{mL}$ and $0.033 \mu \mathrm{g} / \mathrm{mL}$, respectively (Goktepe \& Plhak 2002). Maranho et al. (2014) observed that the neem oil based biopesticide showed an $\mathrm{EC}_{50}$ of $0.17 \mathrm{ml} / \mathrm{L}$ for $D$. magna. Comparison of the $\mathrm{LC}_{50}$ values obtained from the previous studies of other neem-based pesticides shows that the $\mathrm{LC}_{50}$ value of Neemark from the present study is very high. This could be attributed to the high range selected for the study along with the possible indication of Neemark being less toxic to the invertebrate aquatic fauna as compared to other neem derived products. Other biopesticides have also shown comparatively high toxicity as compared to Neemark. Camphor, a major ingredient of biopesticides, was assessed for its acute toxicity to D. magna (Yim et al. 2014). In their study, they observed immobilization of $D$. magna over $98.8 \mu \mathrm{M}$ and the $\mathrm{EC}_{50}$ was found to be $395 \mu \mathrm{M}$ after $48 \mathrm{~h}$. 
The median lethal concentration for Tafgor for Series 1 (100-1000 ppm) and Series 2 (1000-4000 ppm) was found to be $439.46 \mathrm{ppm}$ and $1335.97 \mathrm{ppm}$, respectively. The $\mathrm{LC}_{50}$ values for other organophosphate pesticides, such as chlorpyrifos and malathion for Daphnia magna were found to be $0.001 \mu \mathrm{g} / \mathrm{mL}$ and $0.033 \mu \mathrm{g} / \mathrm{mL}$, respectively by Leight \& Dolah (1999). Several other studies for the $\mathrm{LC}_{50}$ of Chloropyrifos on D. magna were conducted which gave the results as follows; $0.19 \mu \mathrm{g} / \mathrm{mL}$ (Kikuchi et al. 2000) and $1.0 \mu \mathrm{g} / \mathrm{mL}$ (Kersting \& van Wijngaarden 1992). There are a few acute toxicity studies of Tafgor conducted on Daphnia. As per the EPA report (US EPA 1983), the $48 \mathrm{~h}$ $\mathrm{LC}_{50}$ value for Tafgor in D. magna is $2.5 \mu \mathrm{g} / \mathrm{mL}$. Various studies have assessed the toxic effects of other chemical groups of insecticides on Daphnia. The $\mathrm{LC}_{50}$ values for carbaryl on Daphnia pulex were found to be $50.1 \mu \mathrm{g} / \mathrm{L}$, $33.1 \mu \mathrm{g} / \mathrm{L}$, and $26.4 \mu \mathrm{g} / \mathrm{L}$ for 24,48 and $72 \mathrm{~h}$, respectively, and $1.5 \mathrm{mg} / \mathrm{L}$ and $1.09 \mathrm{mg} / \mathrm{L}$ for 24 and $48 \mathrm{~h}$, respectively for imidacloprid (Hassoon \& Salman 2016). However, the $\mathrm{LC}_{50}$ values were higher when D. magna were exposed to imidacloprid with concentrations going beyond $102000 \mu \mathrm{g} / \mathrm{L}$ for $48 \mathrm{~h}$ (Raby et al. 2018). This suggests that variation in the threshold to imidacloprid can occur in different species of Daphnia. A study in Turkey showed that the acute toxic lethal concentration values for Abamectin, a chemical insecticide, were $0.02 \mu \mathrm{g} / \mathrm{L}$ and $0.0043 \mu \mathrm{g} / \mathrm{L}$ for 24 and $48 \mathrm{~h}$, respectively (Azgin \& Goksu 2016). A study to examine the effects of fungicides on D. pulex in Chile revealed that the $\mathrm{LC}_{50}$ of carbendazim was $26.1 \mu \mathrm{g} / \mathrm{L}$ (Encina et al. 2017). In the present study, Daphnia showed a relatively higher tolerance to Tafgor which could be due to the lower exposure time as opposed to the other reference studies where the exposure time was greater and thus, the subsequent $\mathrm{LC}_{50}$ values were comparatively lower than Tafgor. Thiamethoxam is an insecticide belonging to the class of neonicotinoids, which is used in

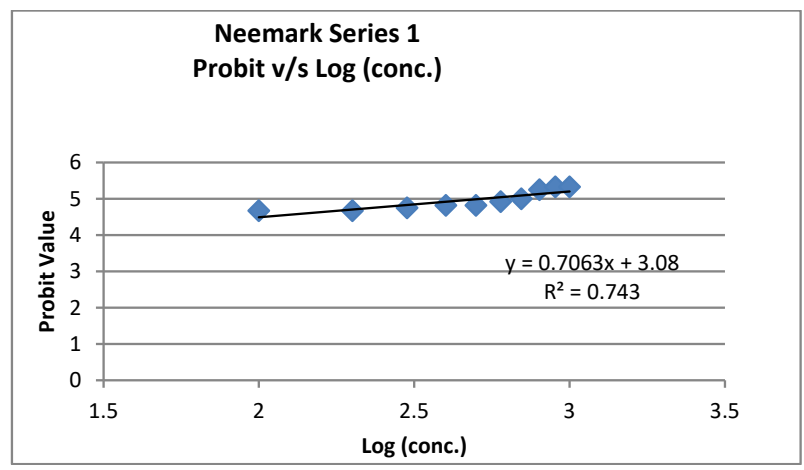

Fig. 1: Effect of varying concentrations of Neemark Series 1 (100-1000 ppm) on the Probit. agriculture to control a broad range of insect pests. The acute toxicity of these pesticides was examined for different aquatic organisms. The results showed that the organisms were least sensitive with acute median effective concentrations greater than $80 \mathrm{mg} / \mathrm{L}$. The $\mathrm{EC}_{50}$ for Daphnia magna for 24 and $48 \mathrm{~h}$ exceeded $100 \mathrm{mg} / \mathrm{L}$ (Finnegan et al. 2017). The acute toxicity of Thiamethoxam on D. magna showed an $\mathrm{LC}_{50}$ value to exceed $80000 \mu \mathrm{g} / \mathrm{L}$ for $48 \mathrm{~h}$ implying higher tolerance to the insecticide (Raby et al. 2018). Neonicotinoids are chemically similar to the natural component nicotine. The literature assessed for the toxicity of thiamethoxam shows that it consequently poses less risk to the aquatic organisms. This could suggest future directives to promote the use of similar types of pesticides imitating natural components or use the biological components completely (Finnegan et al. 2017). The results of the median lethal concentration obtained for Neemark and Tafgor showed that Tafgor is comparatively more lethal to Daphnia than Neemark and thus, showing more toxicity. This also indicates that the current dosage of Tafgor of $2500 \mathrm{ppm}$ used on the field by farmers may be highly toxic to the aquatic fauna. The current dose of Neemark that is used on the field i.e. 500 ppm may be comparatively less toxic to the aquatic fauna as compared to Tafgor. The percent mortality for the two pesticides for the respective series was converted into probit values using the probit table and the concentrations were converted into log (concentration) values. The corresponding findings (Figs. 1-4) indicate that there is an increase in the probit value with the increase in the pesticide concentration. The probit values for Series 1 of Neemark and Tafgor show a similar mortality trend (Figs. 1 and 3). In contrast, the percentage mortality for Tafgor Series 2 shows close to $100 \%$ mortality at probit values of 8.09 for the last two $\log$ (conc.) i.e. for $3500 \mathrm{ppm}$ and 4000 ppm (Fig. 4). This trend was absent in the Neemark Series 2, which shows maximum mortality of $73 \%$ at probit 5.61 (Fig. 2).

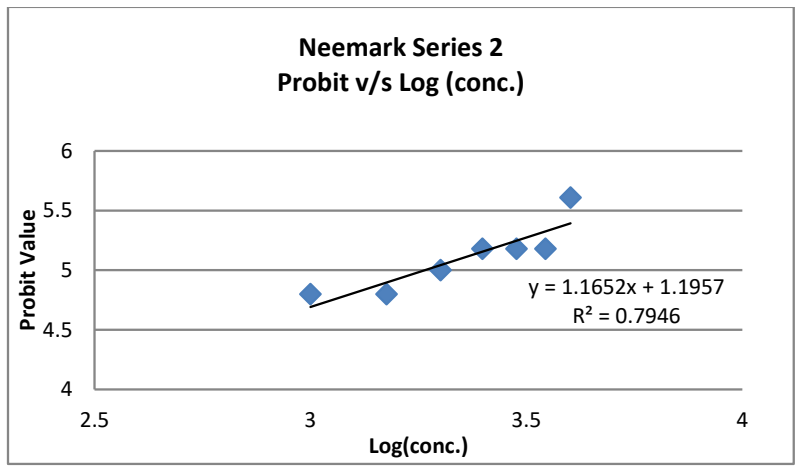

Fig. 2: Effect of varying concentrations of Neemark Series 2 (1000-4000 ppm) on the Probit. 


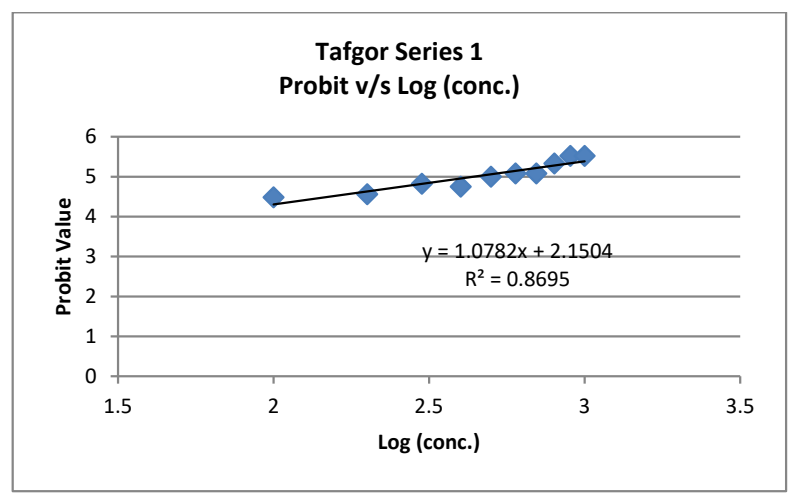

Fig. 3: Effect of varying concentrations of Tafgor Series 1 (100-1000 ppm) on the Probit.

An overall comparison of the average percentage mortality of the two series of Neemark and Tafgor against different pesticide concentrations showed that the percentage mortality of Tafgor remained, in general, greater than that of Neemark (Figs. 5 and 6).

This highlights the possible subsequent negative implications of Tafgor on aquatic life when used on fields due to its highly toxic chemical composition. The concentrations of Neemark used, which are the same as that of Tafgor however, shows comparatively lower mortality rates compared to those of Tafgor and thus, subsequently pointing to lesser negative effects on the aquatic fauna.

\section{Combination Ratio Treatment}

As some of the farmers use a combination of Neemark and Tafgor, the in vitro combination ratio treatment of Neemark and Tafgor was done to find a suitable ratio treatment that would cause minimum harm to the aquatic fauna. The highest percentage mortality (50\%) and the corresponding lowest $\mathrm{LC}_{50}$ value $(255.86 \mathrm{ppm})$ was seen in the 1:1 (Neemark:

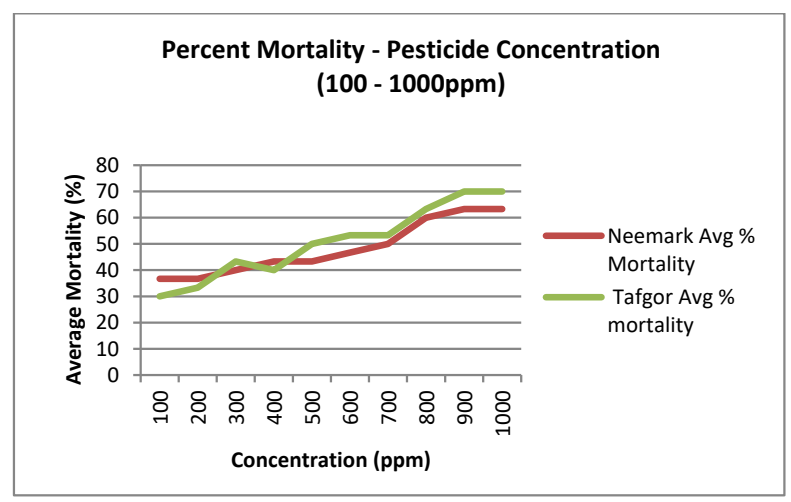

Fig. 5: Comparison of average percentage mortality for series 1 (100$1000 \mathrm{ppm}$ ) of Neemark and Tafgor.

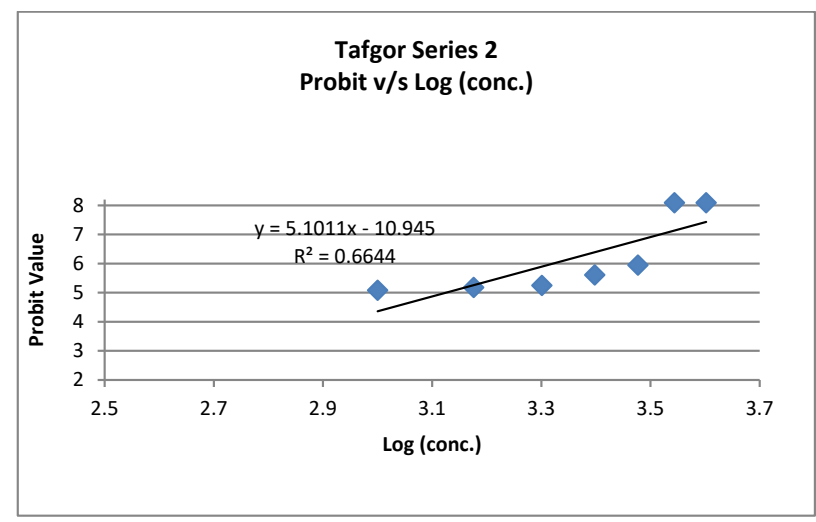

Fig. 4: Effect of varying concentrations of Tafgor Series 2 (1000-4000 ppm) on the Probit.

Tafgor) ratio treatment. However, the median percentage mortality was $40 \%$ for both 1:2 (Neemark: Tafgor) and 2:1 (Neemark: Tafgor) ratios and the corresponding $\mathrm{LC}_{50}$ values for the 1:2 and 2:1 ratio treatment were $427.43 \mathrm{ppm}$ and $1067.78 \mathrm{ppm}$, respectively. The median percentage of mortality values have been depicted in Fig. 7. The least lethal treatment was found to be the $2: 1 \mathrm{v} / \mathrm{v}$ ratio treatment, which suggests that that combination treatment with two volumes of Neemark against one volume of Tafgor is the preferred treatment if a combination of Neemark and Tafgor are to be used on the field as Neemark is a natural ingredient product with low ill effects on non-target fauna. However, the efficiency of this pesticide used in killing the pest organisms needs further assessment.

Two-way ANOVA was carried out to assess whether the different concentrations and the combination ratio treatment of Neemark and Tafgor affected the percentage mortality significantly or not. To the best knowledge of the authors, two-way ANOVA is being used for the first time to study the impact of concentration and combination ratio treatments on Daphnia's mortality. The percent mortality showed a

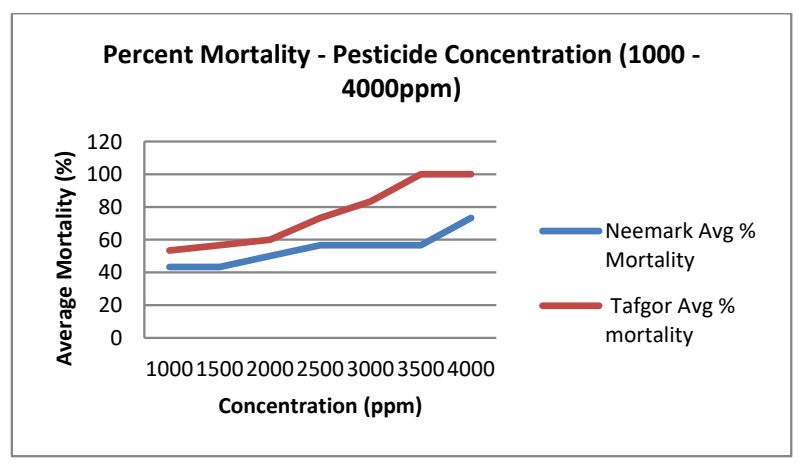

Fig. 6: Comparison of average percentage mortality for series 2 (1000$4000 \mathrm{ppm}$ ) of Neemark and Tafgor. 


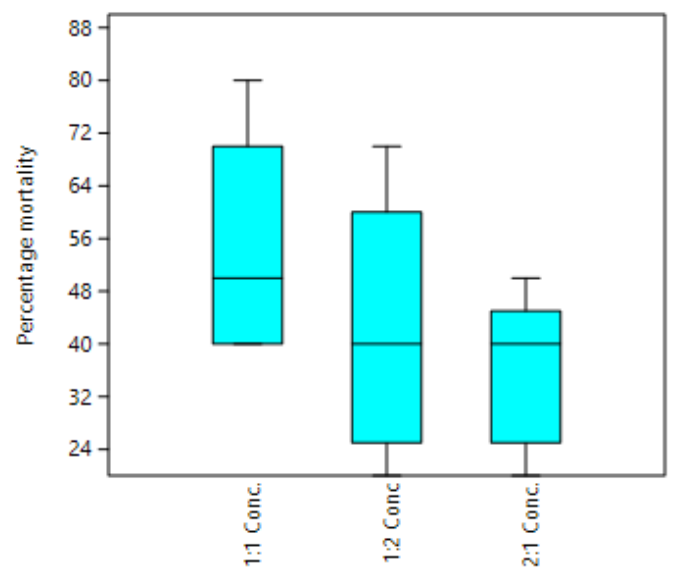

Fig. 7: Boxplot showing the median percent mortality of Daphnia.

significant difference for different combination treatments $(\mathrm{F}=7.166, \mathrm{p}<0.05)$ and the different concentrations $(\mathrm{F}$ $=19.729, \mathrm{p}<0.05)$ but the combined effect of both the concentration and the combination treatment showed no significant effect on the percentage mortality. The results are shown in Table 1.

The statistical t-test has been used for the first time to compare and check for the significant difference in the mortality rates of Daphnia magna between the two pesticides. Unpaired t-test was applied to check whether the percentage mean mortality of Series 2 (1000 ppm-4000 ppm) of Tafgor showed a significant difference from the Series 2 (1000 ppm$4000 \mathrm{ppm}$ ) of Neemark and a significant difference was seen $(\mathrm{p}<0.05, \mathrm{p}=0.034, \mathrm{t}=2.483)$. No significant statistical difference was seen between the two series of Tafgor and Neemark of the range $100 \mathrm{ppm}$ to $1000 \mathrm{ppm}$ ( $\mathrm{p}>0.05)$. Thus, the concentration of Tafgor could be used within the concentration of $1000 \mathrm{ppm}$ on the field by the farmers and still not show much difference in the death of the Daphnia when compared to Neemark. However, increasing the concentration of the two pesticides above $1000 \mathrm{ppm}$ would cause a significant effect on the mortality rates with the possibility of Tafgor showing more lethality to the aquatic fauna. This

Table 1: Results for Two-way ANOVA analysis.

\begin{tabular}{|llll|}
\hline & F value & P value & F critical \\
\hline $\begin{array}{l}\text { Combination Ratio Treat- } \\
\text { ment }\end{array}$ & 7.1666 & $0.006538^{*}$ & 3.68232 \\
$\begin{array}{l}\text { Pesticide Concentration } \\
\text { (ppm) }\end{array}$ & 19.7291 & $7.74 \mathrm{E}-06^{*}$ & 3.055568 \\
$\begin{array}{l}\text { Interaction between combi- } \\
\text { nation ratio treatment and } \\
\text { pesticide concentration }\end{array}$ & 1.3854 & 0.278997 & 2.640797 \\
\hline
\end{tabular}

*significant at $\mathrm{p}=0.05$ suggests that the current dosage concentration of $2500 \mathrm{ppm}$ may show more toxic effects on the aquatic fauna.

\section{CONCLUSION}

Non-target toxicity of pesticides on the aquatic fauna stands to be an important environmental concern. The $\mathrm{LC}_{50}$ values of Neemark and Tafgor pesticides showed that the chemical pesticide, Tafgor, was comparatively more toxic to Daphnia than the biological counterpart, Neemark. A greater proportion of farmers used either only biopesticides or a mixture of biopesticide and chemical pesticide. In comparison, a few of the surveyed farmers used only chemical pesticides in their fields. Further reduction in this proportion would be possible with more awareness among the farmers backed up with scientific study.

The initial evaluation of the combination ratio treatments suggests that if both the types of pesticides need to be used for better protection against pests, then the most preferable treatment would be to use a mixture of two volumes of Neemark with one volume of Tafgor. This combination exhibits the lowest toxicity to Daphnia as opposed to $1: 1 \mathrm{v} / \mathrm{v}$ or 1:2 v/v of Neemark and Tafgor. Our study has shown that concentration and the combination ratio treatment critically affect the percent mortality of Daphnia. Thus, these can act as governing factors that can be considered, when using a combination of these pesticides on the field which would, in turn, ensure a healthy aquatic environment. The efficiency of these pesticides, with varying concentrations and combination treatments, to eradicate the target pest organisms needs further assessment which would strengthen their application on the field. This would not only promote good crop and soil health but also facilitate a healthy aquatic ecosystem sustainable for other aquatic life forms.

\section{REFERENCES}

Azgin, C. and Goksu, M. Z. L. 2016. Acute toxicity of Abamectin (insecticide) on Daphnia magna (Straus, 1820). Journal of Biological and Environmental Science, 10(30): 125-128.

Bharadwaj, T. and Sharma, J. P. 2013. Impact of pesticides application in agricultural industry: an Indian scenario. International Journal of Agriculture and Food Science Technology, 4(8): 817-822.

Demetrio, P. M., Bonetto, C. and Ronco, A. E. 2014. The effect of cypermethrin, chlorpyrifos, and glyphosate active ingredients and formulations on Daphnia magna (Straus). Bulletin of Environmental Contamination and Toxicology, 93: 268-273.

Dodson, S. I., Merritt, C. M., Torrentera, L., Winter, K. M., Tornehl, C. K. and Girvin, K. 1999. Dieldrin reduces male production and sex ratio in Daphnia galeata mendotae. Toxicology and Industrial Health, 15: 192-199.

Eldridge, B. F. 2008. Pesticide Application and Safety Training for Applicators of Public Health Pesticides. Sacramento: California Department of Public Health, Vector-Borne Disease Section. 
Encina, F., Escalante, P. de L. R. and Salazar, K. 2017. Acute toxicity $\left(\mathrm{LC}_{50}\right)$ of a pesticide (Carbendazim) on two native crustacean zooplankton species: Daphnia pulex Leidig, 1860 and Tumeodiaptomus diabolicus (Brehm 1935) from northern Patagonian lakes (Chile). Crustaceana, 90(2): 199-206.

Finnegan, M. S., Baxter, L. R., Maul, J., Hanson, M. L. and Hoekstra, P. F. 2017. Comprehensive characterization of the acute and chronic toxicity of the neonicotinoid insecticide Thiamethoxam to a suite of aquatic primary producers, invertebrates and fish. Environmental Toxicology and Chemistry, 36(10): 2838-2848.

Finney, D. J. 1971. Probit Analysis 3rd ed. Cambridge University Press, London.UK.

Goktepe, I. and Plhak, L. C. 2002. Compartive toxicity of two azadirachtin - based neem pesticides to Daphnia pulex. Environmental Toxicology and Chemistry, 21(1): 31-36.

Hanazato, T. 2000. Pesticide effects on freshwater zooplankto: an ecological perspective. Environmental Pollution, 112: 1-10.

Hassoon, H. A. and Salman, S. A. 2016. The acute effect of pesticides Carbaryl and Imidacloprid on Daphnia pulex species. Journal of International Environmental Application and Science, 11(1): 18-28.

Hummel, H. E., Hein, D. F. and Schmutterer, H. 2012. The coming of age of azadirachtins and related tetranortriterpenoids. Journal of Biopesticides, 5: 82-87.

Kandpal, V. 2014. Biopesticides. International Journal of Environmental Research and Development, 4: 191-196.

Kekane, A. 2013. Indian agriculture - status, importance and role in Indian economy. International Journal of Agriculture and Food Science Technology, 4(4): 344 - 346.

Kersting, K. and van Wijngaarden, R. 1992. Effect of chloropyrifos on a microecosystem. Environmental Toxicology and Chemistry, 11: 365-372.

Kikuchi, M., Sasaki, Y. and Wakabayashi, M. 2000. Screening of organophosphate insecticide pollution in water by using Daphnia magna. Ecotoxicology and Environmental Safety, 47(3): 239-245.

Kumar, S. 2012. Biopesticides: A need for food and environmental safety. Journal of Biofertilizers and Biopesticides, 3(4): 1-3.

Kumar, S. and Singh, A. 2015. Biopesticides: Present status and the future prospects. Journal of Fertilizers and Pesticides, 6(2): 1-2.

Leight, A. K. and Dolah, R. F. V. 1999. Acute toxicity of the insecticides endosulphan, chloropyrifos and malathion to the epibenthic estuarine amphipod Gammarus palustris(Bousfeild). Environmental Toxicology and Chemistry, 18(5): 958-964.

Luo, Y., Zang, Y., Zhong, Y. and Kong, Z. 1999. Toxicological study of two novel pesticides on earthworm Eisenia foetida. Chemosphere, 39(13): 2347-2356.

Maranho, L. A., Botelho, R. G., Inafuku, M. M., de A.R. Nogueira, L., de Olinda, R. A., de Sousa, B. A. and Tornisielo, V. L. 2014. Testing the neem biopesticide (Azadirachta indica A. Juss) for acute toxicity with Danio rerio and for chronic toxicity with Daphnia magna. Journal of Agricultural Science and Technology, 16: 105-111.

Marrone, P. G. 2014. The market and potential for biopesticides. In: Coates, et al. Biopesticides: State of the Art and Future Opportunities. Proceedings of the American Chemical Society Symposium Series.

Pimentel, D., Acquay, H., Biltonen, M., Rice, P., Silva, M., Nelson, J., Lipner, V., Giordano, S., Horowitz, A. and D' Amore, M. 1992. Environmental and economic costs of pesticide use. BioScience, 42(10): 750-760.

Raby, M., Nowierski, M., Perlov, D., Zhao, X., Hao, C., Poirier, D. G. and Sibley, P. K. 2018. Acute toxicity of six neonicotinoid insecticides to freshwater invertebrates. Environmental Toxicology and Chemistry, 37(5): 1430-1445.

Saucke, H. and Schmutterer, H. 1992. Investigations on side-effects of various neem products on Daphnia magna Straus (Crustacea: Cladocera). Journal of Pest Science, 65: 121-126.

Scott, I. M. and Kaushik, N. K. 1998. The toxicity of Margosan-O, a product of neem seeds, to selected target and nontarget aquatic invertebrates. Archives of Environmental Contamination and Toxicology, 35: 426-431.

Stark, J. D. 2001. Population-level effects of the neem insecticide, Neemix, on Daphnia pulex. Journal of Environmental Science and Health, B36(4): 457-465.

Stark, J. D. 2007. Ecotoxicology of neem. ACS Symposium Series, 947 : 275-286.

Subash, P.S., Prem Chand, S., Pavithra, J. B. S. and Pal, S. 2017. Pesticide use in Indian agriculture: trends, market structure and policy. ICAR - National Institute of Agricultural Economics and Policy Research, 1-4.

U.S. Environment Protection Agency (EPA). Fate and environmental risk assessment chapter. 2000. Available online: http://www.epa.gov/ pesticides/op/chlorpyrifos/efedrra1.pdf. Accessed on $5^{\text {th }}$ September 2019.

U.S. Environmental Protection Agency. 1983. Dimethoate: Proposed Tolerance. Federal Register 48.

Yadav, I. C. and Devi, N. L. 2017. Pesticides classification and its impact on human and environment. Environmental Science and Engineering, 6: $140-158$

Yadav, I. C., Devi, N. L., Syed, J. H., Cheng, Z., Li, J., Zhang, G. and Jones, K. C. 2015. Current status of persistent organic pesticides residues in air, water, and soil, and their possible effect on neighboring countries: A comprehensive review of India. Science of the Total Environment, 511: 123-127.

Yim, E. C., Kim, H. J. and Kim, S. J. 2014. Acute toxicity assessment of camphor in bio-pesticides by using Daphnia magna and Danio rerio. Environmental Health and Toxicology, 29: 1-8. 